Revista Sostenibilidad, Tecnología y Humanismo

54

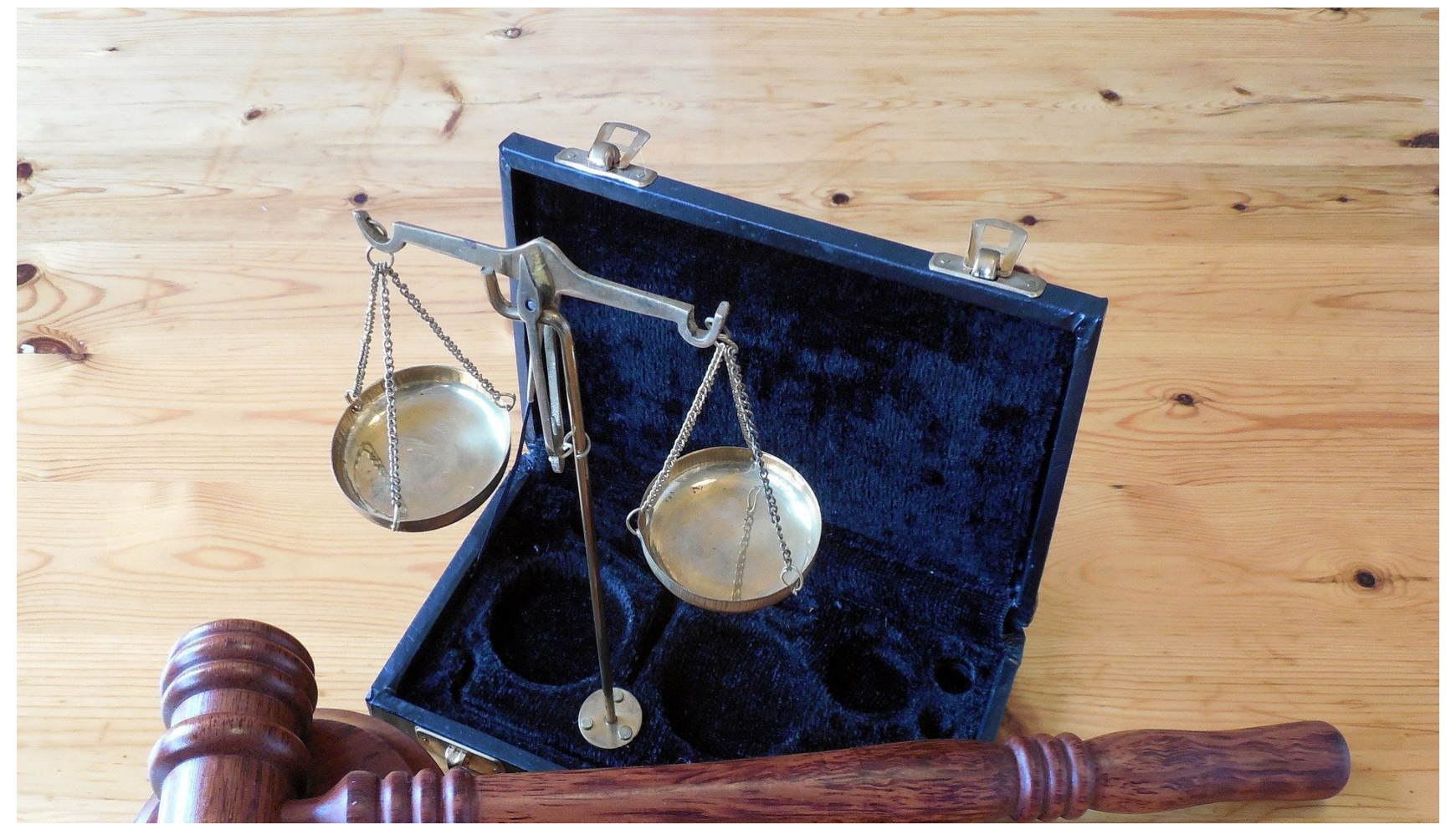

\title{
El derecho como creación autopoética. análisis desde la perspectiva de Günther Teubner
}

\section{Law as an autopoetic creation. analysis from the perspective of Günther Teubner}

María Alejandra Benítez-Hurtado

Magíster en Derecho Administrativo, alejandra.benitez94@hotmail.com, Fundación Tecnológica Antonio de Arévalo - Tecnar, Cartagenda de Indias, Colombia 
Resumen

El presente trabajo explora los rasgos fundamentales de la denominada creación autopoiética en el derecho, concibiéndolo como un producto cultural, a partir de las aportaciones del profesor Günter Teubner. Sus reflexiones apuntan a que los componentes del Derecho constituyen con sus interacciones la red de producción que los origina, es decir, que el Derecho sólo crea a partir del Derecho. Lo anterior trae consigo varias consecuencias: el abandono de la "norma" como objeto del derecho y que se dirija la mirada hacia las interdependencias y comunicaciones -sustento lingüístico- que se dan en un espacio edificado y demarcado por ellas, aunque en el plano ontológico no existen, además, que se abandone la percepción del derecho como pretendiente de la dirección de la orquesta social, y más bien se piense en un derecho reflexivo, con el fin de hacerle contrapeso a su materialización y a la autonomía de la sociedad en ciertas áreas.

Palabras clave: Autopoiesis, Constructivismo Epistemológico, Comunicación Social, Autorreferencialidad, Trampa Epistémica, Derecho Reflexivo.

\section{Abstract}

This paper explores the fundamental features of the so-called autopoietic creation in law, conceiving it as a cultural product, based on the contributions of Professor Günter Teubner. His reflections point to the fact that the components of Law constitute, with their interactions, the production network that originates them, that is, that Law only creates from Law. The foregoing brings with it several consequences: the abandonment of the "norm" as an object of law and that the gaze be directed towards the interdependencies and communications linguistic sustenance - that occur in a space built and demarcated by them, although on the ontological level Furthermore, there is no abandonment of the perception of law as a pretender to the leadership of the social orchestra, but rather a reflexive law, in order to counterbalance its materialization and the autonomy of society in certain areas.

Keywords: Autopoiesis, Constructivism Epistemological, Social Communication, selfreferentiality, Epistemic Trap, Reflexive law. 


\section{6}

\section{Introducción}

La noción de Sistema se constituye como el nuevo paradigma en la ciencia planteado en este contexto. Siguiendo a Ramírez del Valle, quien cita a Bertalanffy, se puede definir sistema como un conjunto de elementos en interacción [1], observándose como elemento esencial la reciprocidad. Mientras que el objeto clásico de la ciencia y del Derecho ha sido la "parte" o la "norma", para los modelos sistémicos lo preponderante es el análisis de las interdependencias y comunicaciones que se dan en un mundo construido y perfilado por ellas, de tal forma que el mundo ya no está compuesto de elementos individuales, sino de interacciones, las cuales se alzan con el nuevo sujeto epistémico.

Son dos vías específicas para abordar la noción de sistema: la primera es la noción de retroalimentación, en que las entradas y las salidas del sistema lo alimentan, reduciendo - amplificando las nociones de este -sistemas abiertos-; la segunda es la noción de autopoiesis, visto el sistema como un conjunto de interacciones que se generan a sí mismas sin contacto con el entorno -sistemas cerrados- [2]. Este estudio versará sobre la segunda noción.

A partir de las críticas a la sociología empírica del Derecho por su falta de nivel para asimilar la complejidad de las sociedades actuales, al advertir sobre las falencias en expresar esa realidad, Teubner propone la concepción del derecho como un sistema íntegramente cerrado en la sociedad - 0 autopoiesis-, el cual adolece de entradas y salidas, con la etiqueta de la autorreferencialidad. Esta concepción nos traslada al abandono de un derecho que "invade" muchas de las esferas del sistema social.

Con la noción de Autopoiesis, para el caso del derecho, la norma jurídica se transforma en un artefacto lingüístico: la norma jurídica ya no es quien crea el derecho, sino que el permanente curso de comunicaciones dentro del sistema es lo que crea la Constitución, la ley, los contratos, etc. Otra de las características del derecho gracias al posicionamiento de este nuevo paradigma es la autorreferencialidad, lo cual se traduce en que las transformaciones que sufre el Derecho no son causadas por factores externos., sino que, bajo sus propios parámetros, reformula o reduce los conflictos que nacen en otros sistemas jurídicos.

Desde la autopoiesis biológica a la autopoiesis social: maturana, varela y otros antecedentes.

El concepto de Autopoiesis tiene su origen en la teoría biológica de Maturana y Varela. La etimología de la palabra deriva del griego autós (por sí mismo) y poiesis (creación, producción). En estricto sentido, significa la capacidad de los sistemas de producirse a sí mismos, que el respectivo sistema es construido por los propios elementos que él construye [3].

Los seres vivos son sistemas determinados por su estructura, es decir, todo lo que ocurre en nosotros, sobreviene en la forma de cambios estructurales determinados en nuestra estructura, ya sean mutaciones propias o acontecidas en nuestras interacciones en el medio, pero no determinados por este. Por ello, este fenómeno postula que los seres vivos somos seres que nos autoproducimos, ya que creamos nuestros componentes en base a los nuestros y a las sustancias que tomamos del entorno. Esta cualidad que se pierde al momento del cese de nuestros signos vitales. La Autopoiesis es, desde la óptica biológica, una red de procesos de producción, evolución y reducción de elementos que, a través de sus interacciones y transformaciones, regeneran y realizan continuamente esa misma red de procesos. No se profundizará más sobre este tema debido a que se desviaría del camino 
demarcado en este estudio, pero lo elemental en este acápite es que la idea de la autopoiesis es aplicable a cualquier sistema y, por ende, al fenómeno social, trasladándose entonces a la recepción del mismo a las ciencias sociales gracias al alemán Niklas Luhmann, quien influenció notablemente en múltiples teorías jurídicas, entre las que se apuntan las tesis en Derecho Penal de Imputación Objetiva y funcionalismo en Günter Jakobs. Teubner, el autor que se estudia toma de Luhmann muchos conceptos, lo cual lo hace deudor de este sociólogo.

Un suceso histórico alimentó notablemente la discusión sobre la teoría de la Autopoiesis, delimitándola aún más, fue un descubrimiento a finales de los años 20 del siglo pasado: el Teorema de Gödel. Matemáticamente, este Teorema ratificó un aspecto esencial en los límites del saber humano, la razón humana no puede explicar ciertas cosas, si no se aceptan que son, no se puede organizar lo que se conoce. Lo anterior encuentra su justificación en la noción de dogmática jurídica: la raíz misma de la denominación nos apunta a la concepción de verdades que provienen de una deidad y que son indiscutibles por regla general, ya que son postulados que no pueden dejar de existir. Un claro ejemplo de la anterior premisa es la figura del Contrato Social: no se puede probar o demostrar que dicho pacto sucedió, es una ficción jurídica, pero si se destruye dicha ilusión, se desarticula el sistema liberal. Con este historial, se dieron los primeros asomos por la ventana jurídica el teatro de la autopoiesis, acompañado de sus personajes que sólo existen en él gracias a las comunicaciones normativas.

Las debilidades de las nuevas teorías de la sociología jurídica: las "trampas" de la autorreferencialidad

Como se precisó en la parte introductoria, se sugiere dar una explicación del derecho dentro de un modelo que concibe al mismo como un sistema autopoiético y autorreferente, al tomar como marco teórico a Günter Teubner.

Este autor, antes de exponer sin obstrucciones su concepción del Derecho como sujeto epistémico, recorre el camino de las nuevas teorías denominadas post-estructuralismo, la teoría crítica, la teoría del discurso y de la autopoiesis, esgrimiendo que su formulación ha provocado lo que él mismo ha denominado Galimatazos o Jabberwocky, ya que antes existía el consenso de que a través de una observación paciente del derecho real en el mundo existente, se lograría un cuerpo de teorías que no serían ni especulativas ni metafísicas [4].

La primera de las teorías que critica Teubner es la planteada por Habermas. De este teórico rescata su rechazo de la noción de verdad como correspondencia y su propuesta de la verdad como consenso, que se declara como "potencial" para distinguir entre lo verdadero y lo falso. Su filosofía ya no le atribuye la autoridad epistémica al sujeto en su autonomía, sino a la comunidad comunicativa: muchos críticos hablan de que en Habermas hay un cambio de paradigma del conocimiento: el conocimiento no es algo externo que hay que captar, sino algo que está dentro de nosotros mismos y que hemos de construir, y reconoce también que el conocimiento es un proceso comunicativo; no en vano, se afirma que la "intersubjetividad" toma el puesto del sujeto epistémico kantiano. Es el auténtico consenso de la comunidad el que determina la verdad en cuestiones cognitivas y normativas [4].

Con la reformulación de mucha de las posturas kantianas, el nuevo a priori está representado por la "situación ideal del discurso" cuyo presupuesto es condición para la posibilidad de comunicación. Desde aquí comienzan los problemas con Habermas: Desgraciadamente este "a priori de la comunidad comunicativa" es 


\section{8}

al mismo tiempo uno de los mayores problemas de esta teoría. Con la apriorización de ciertas características de la comunicación, Habermas pretende escapar de las «paradojas de la autoreferencia» que necesariamente surgen de su jerarquía de justificación discursiva [4] Indica Teubner:

El núcleo de la teoría de Habermas se encuentra en la auto-aplicación de las prácticas discursivas: los procedimientos del discurso sólo pueden ser justificados por el propio discurso, cuyos procedimientos tienen a su vez que ser justificados por el discurso. (Con el fin de evitar la regresión infinita y la circularidad, Habermas recurre al trascendentalismo comunicativo. La fundamentación trascendentalista del discurso racional se encuentra en estrecha vinculación con las ambigüedades de la «intersubjetividad». Éstas representan el otro gran problema no resuelto en la posición de Habermas sobre el conocimiento social. ¿Qué es lo que se pretende: elementos o relación? ¿Conciencia o comunicación? ¿Procesos psíquicos o sociales? [4].

Habermas intenta esquivar a como dé lugar las trampas epistémicas de la autorreferencialidad; su argumento se cae con una simple pregunta: ¿Cómo puede justificarse el discurso racional sino por el propio discurso racional?

Otro de los autores citados por Teubner es Michel Foucault, famosos por sus ideas el discurso y la episteme, vistas como una radicalización de las ideas del anterior teórico. El punto de arranque de toda su teoría es constructivista: la realidad no es algo externo al conocimiento, sino que es construido por el conocimiento en sí. Asevera que la conciencia individual no construye la realidad, ni mucho menos la intersubjetividad, si no que la realidad es el producto de la interacción entre actores humanos.
El sujeto humano ya no es el autor del discurso, es el discurso quien produce al sujeto humano como un artefacto semántico y formaciones discursivas son históricamente contingentes, debido a su carencia de fundamentación a priori.

De la anterior idea es que se sobrevienen sus debilidades: Foucault evita a toda costa las necesarias consecuencias de su propia construcción e introduce el concepto de poder, con el fin de exteriorizar las relaciones autorreferenciales. Termina cayendo nuevamente, al igual que Habermas, en las trampas de la autorreferencialidad: lo anterior se ve manifiesta ante la imposibilidad de la Teoría de Foucault de responder a lo siguiente: ¿Cómo pueden ser transformadas esas formaciones discursivas que gobiernan el epistème de toda una época histórica sino por las propias formaciones discursivas?

En vista de lo anterior, aparece Niklas Luhmann, quien concibe la sociedad como "funcionalestructural", viendo el Sistema Social como un ente móvil, como un juego de antinomias interior-exterior, identidad-diferencia, que buscan, entre otras cosas, mantener su unidad, atacada por las presiones de los estímulos que provienen de su medio.

Opina que las paradojas 0 trampas epistémicas de la autorreferencialidad son el principal obstáculo para el desarrollo de una epistemología social; afirma que las paradojas no pueden evitarse, se deben enfrentar haciendo uso de ellas. Muy sabiamente Teubner asevera:

$\mathrm{Si}$ los discursos sociales son sistemas autopoiéticos, esto es, sistemas que recursivamente producen sus propios elementos gracias a su propia red de elementos, entonces se basan en la autorreferencialidad que tanto Habermas como Foucault intentan desesperadamente evitar. Como sistemas autopoiéticos, los discursos no pueden sino 
justificarse en su propia circularidad y no puede sino producir regularidades que se regulan a sí mismas y que gobiernan la transformación de sus propias regularidades [4].

La paradoja de la Autorreferencia no es entonces un fallo de nuestra reconstrucción intelectual del discurso que hay que evitar a toda costa, sino que es su realidad la que no podemos evitar en modo alguno. La aplicación recursiva de las operaciones a los resultados de estas mismas operaciones no conduce necesariamente al bloque paradójico de la paradoja o a la mera arbitrariedad, sino que, bajo ciertas condiciones, conduce a que surjan los valores propios [4].

De lo anterior, chistosamente, como una comedia o una pantomima, vale decir que el Derecho, la ficción jurídica que estudiamos, está fundado en las paradojas de la Autorreferencialidad.

El problema que debe solucionar la teoría de la autopoiesis -y lo hará Teubner a lo largo de sus tesis-, es sobre cómo tratar las interrelaciones entre las diferentes epistemes autónomas, sus conflictos, incompatibilidades e interferencias. También debe resolver Teubner, después de este paseo sociológico por las teorías que han originado lo que él mismo ha denominado los Galimatazos o Jabberwocky, qué es el Derecho y cómo se concibe como sujeto epistémico [5].

El derecho como hiperciclo autopoiético según la postura de gunther teubner. precisiones $y$ fundamentos.

La teoría de la autopoiesis de Teubner dista considerablemente de las concepciones seculares sobre los sistemas y las organizaciones, afirma que el lenguaje de hoy en día no tiene la prerrogativa ni la facultad de expresar la construcción de realidades sociales cuando éstas son percibidas de manera novedosa [4]. Lo anterior se debe a la multiplicidad de teorías sociológicas enfocadas en lo jurídico, que se trasladan a lo metafísico e ignoran ese derecho real:

1. Las organizaciones no están compuestas por seres humanos como miembros, sino por comunicaciones; concretamente por decisiones, que son sus elementos autoconstitutivos.

2. Las organizaciones "conocen". A través de la comunicación interna construyen sus propias realidades sociales, las cuales difieren considerablemente de las construcciones de la realidad de sus miembros individuales. En pocas palabras: las organizaciones son sujetos epistémicos.

3. Las organizaciones no son capaces per se de acción colectiva. Se transforman a sí mismas en actores colectivos mediante la constitución comunicativa de su identidad.

4. La capacidad de acción colectiva surge cuando las organizaciones, en su identidad colectiva, producen acciones $\mathrm{y}$, viceversa, la acción organizativa produce su identidad colectiva [4].

Sus planteamientos nos dan una primera aproximación, al partir de la anterior noción aplicable a los sistemas sociales, a la situación del derecho, indicándonos que, en este nuevo entorno de sociedades caracterizadas por su complejidad, este debe abandonar su identificación como un simple cuerpo legal o su inmersión en códigos, y considerarse como un sistema compuesto por comunicaciones de índole normativa.

Vale precisar que la creación autopoiética en los fenómenos culturales (como el derecho), se presupone, es un fenómeno lingüístico. Es 


\section{0}

lógico el anterior razonamiento ya que no existe cultura sin lenguaje, el fundamento es la comunicación social.

El docente David Mercado en su Manual de Teoría Constitucional expresa, al tomar como referente la teoría del lenguaje del siglo pasado: cada campo del saber es en sí mismo un lenguaje especial, un modo distinto de emplear el vocabulario de un mismo idioma [6].

En el proceso de la comunicación social se distinguen cuatro fundamentos que se encuentran fuertemente entrelazados: proceso, elemento, estructura e identidad y a partir de ellos el derecho adquiere forma. Teniendo en cuenta el primer elemento, el conflicto es una constante en toda sociedad, con independencia de la ideología y del modo de producción imperante. De acuerdo con el segundo componente, todos los elementos de la comunicación social se nos presentan en forma de actos y hechos. Con referencia al tercer fundamento, toda sociedad tiene normas, bien sean consuetudinarias o escritas; y de acuerdo con el cuarto fundamento, todo fenómeno comunicativo tiene que conocerse a sí mismo como fenómeno comunicacional y se reconoce a sí mismo a través de una representación del universo y esa representación es la concepción del mundo. La parte conflictiva se exterioriza en forma jurídica a partir de los procedimientos legales creados por el derecho para enfrentar los conflictos y solucionarlos; desde el punto de vista elemento, los actos humanos se convierten en actos jurídicos porque el derecho les da esa connotación y luego entonces las normas de conducta social se transforman en normas jurídicas. Lo anterior se grafica de la siguiente forma:

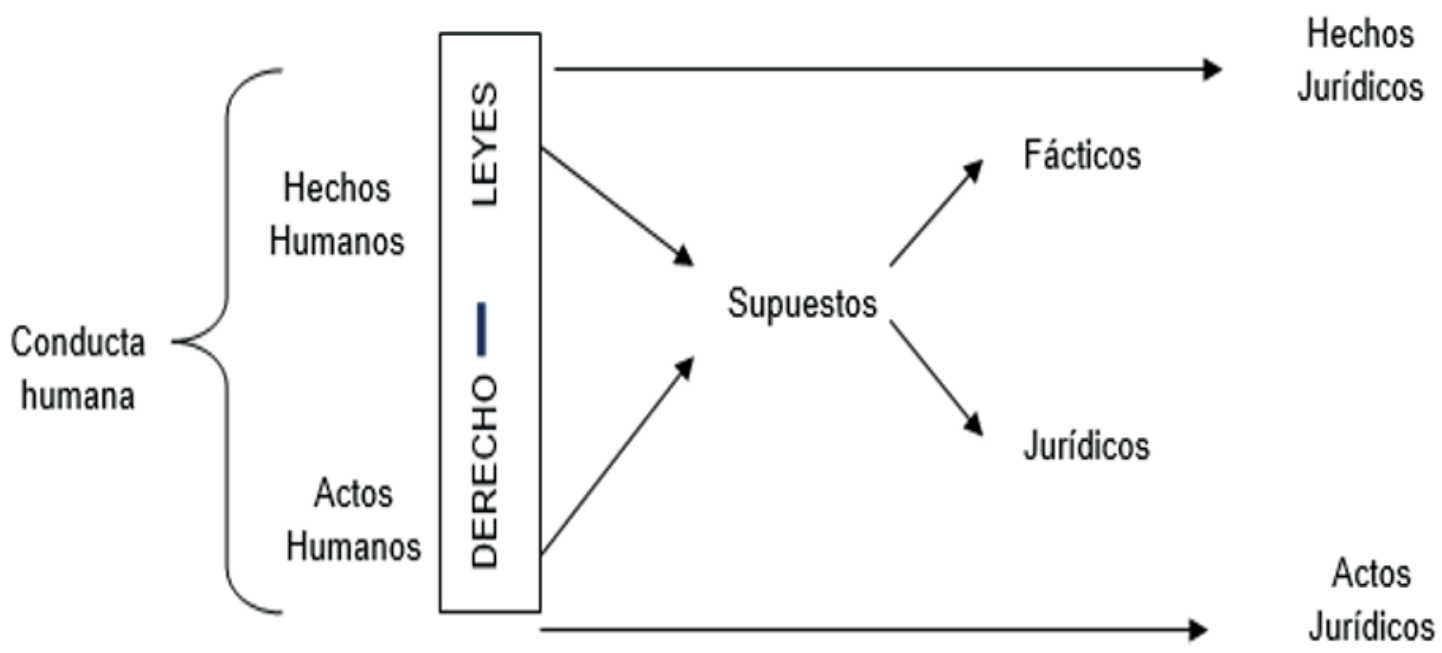

Finalmente, el fundamento identidad, nos da la ideología, una representación del todo contexto de las variables culturales lo cual se traduce en la forma como el derecho va a construir su contenido material, es decir, la doctrina jurídica. Teniendo en cuenta lo anterior, Teubner asevera que el Derecho se inventa a sí mismo, esto nos remite a la noción de autopoieisis.

La autopoiesis en el derecho implica un sistema que produce él mismo su propia estructura y los elementos propios desde los cuales se compone, así el sistema de comunicaciones se produce mediante comunicaciones y, por tanto, autorreferencialmente. De igual forma 
ocurre con todas las operaciones relativas al sistema que se produce en su interior. La siguiente gráfica explica lo dicho en líneas atrás:

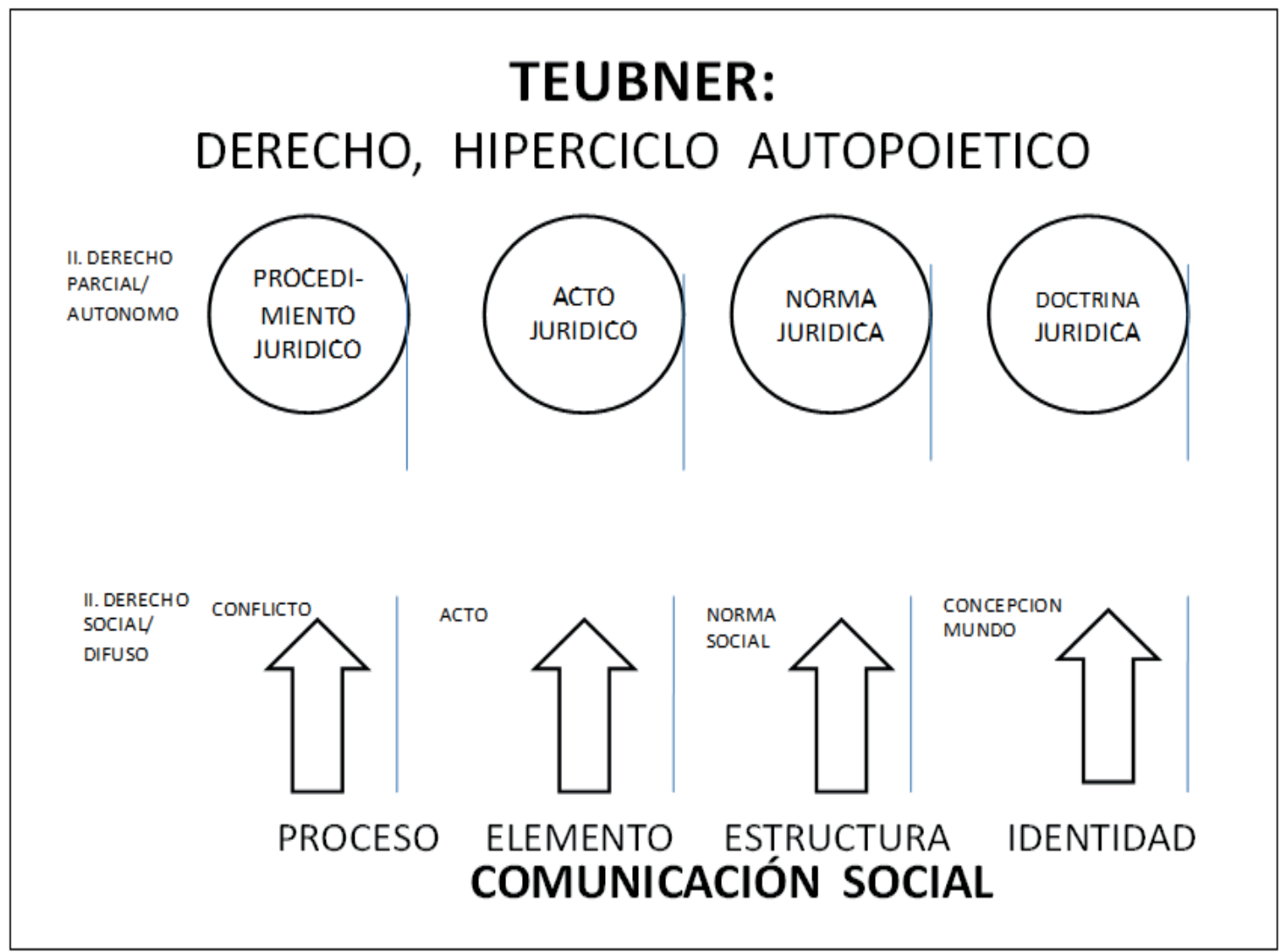

Las ideas base para considerar al derecho como un sujeto epistémico las plasma en su ensayo El Derecho como sujeto epistémico: Hacia una epistemología constructivista del Derecho así:

1. A tenor de la epistemología social constructivista, las percepciones del Derecho respecto de la realidad no se corresponden con algún tipo de realidad social que está «ahí fuera». Por el contrario, es el Derecho el que, como sujeto epistémico autónomo, construye su propia realidad social.

2. No son los seres humanos los que mediante sus acciones intencionales producen el Derecho como un artefacto cultural. Muy al contrario, es el Derecho el que, como proceso comunicativo, crea actores humanos como artefactos semánticos a través de sus operaciones jurídicas.

3. Dado que la sociedad moderna viene caracterizada, de un lado por fragmentarse en diferentes epistèmes y, de otro, por la interferencia entre los mismos, el discurso jurídico se ve atrapado en una «trampa epistémica». La simultaneidad en la dependencia e independencia respecto de los otros discursos sociales es la razón por la cual el Derecho moderno está permanentemente oscilando entre posiciones de autonomía y heteronomía cognitiva [4]. 


\section{2}

Lo anterior es una clara crítica al realismo y propone un constructivismo epistemológico que implique al sujeto en la observación del objeto, que incite a superar la separación de sujeto y objeto para entrar en el concepto de distinción de estos. Las percepciones jurídicas de la realidad son construcciones realizadas por el derecho como sujeto epistémico autónomo, por tanto, los individuos no crean derecho como algo natural, sino que es el derecho como proceso comunicativo el que produce, mediante operaciones jurídicas, los actores humanos como artefactos semánticos. La importancia de que el derecho está compuesto por comunicaciones y no por normas jurídicas radica en evitar la posibilidad de tropezar con el individualismo metodológico, el cual perfilaría al derecho como un cúmulo de normas restrictivas de lo individual, lo cual sólo conduciría al estudio de lo jurídico en su aspecto estático [6].

El tercer postulado de para considerar al derecho como un sujeto epistémico trae a colación la tensión propia de la autonomía dependencia (autonomía y heteronomía) frente a otros discursos de la sociedad: el problema entre autonomía y heteronomía lo centra Teubner en el argumento de que el discurso jurídico es al mismo tiempo un discurso social, como lo son los otros discursos creados por los demás sistemas para el derecho, los cuales están ensimismados en el hiperciclo social. Empero, a pesar de que estos sistemas no se tocan entre sí, si participan del mismo horizonte, lo cual les posibilita la interferencia recíproca, al trabajar sobre un mismo campo de complejidad fenoménica susceptible de reducción. Pero, como esa interferencia recíproca ocasiona una mengua de motivación en otros sectores sociales ya que la validez jurídica lo es exclusivamente para el Derecho, este debe apelar a ciertos mecanismos para lidiar este inconveniente, que pueden ser la sanción o la persuasión, los cuales por no ser lo suficientemente reflexivos, por carecer de la facultad para orientar operativamente a los sistemas en su contingencia, debería ser desestimado por la política de opciones [7].

El derecho, por lo anterior, debe aminorar su poder en ciertos dominios y hacer abandono de sus pretensiones de regulación jerárquica, produciendo una regulación opcional, que los actores requerirán según sus necesidades, es decir, el Derecho dejaría de estar construido sobre la base de expectativas conductuales autoritarias.

Como lo indica Günter Teubner, el sistema jurídico no puede caer en la trampa de querer atrapar la plena autoridad de la construcción de realidades involucradas, sin poder delegar totalmente dicha autoridad a otros discursos sociales, como el político, sino que como condición previa de incorporación de conocimiento social, el derecho individualiza ciertos requisitos básicos referentes al procedimiento y el método cognitivo, es decir, se produce un fenómeno de procedimentalización del sistema jurídico, como mecanismo de coordinación, y de acoplamiento estructural de los otros sistemas entre sí.

Es adecuada la postura de Teubner al abordar el problema autonomía - heteronomía: si se habla de mayor intervención del Estado se introduce disfuncionalidades al sistema, cambios en la función del derecho, en su legitimación y estructura, por ende, el derecho se convierte en un instrumento para la invención política del Estado Social de Derecho. Siguiendo esta línea argumentativa, el derecho deja de ser un instrumento para la solución de conflictos y se alza como un derecho regulatorio; es por eso por lo que el autor estudiado en su otro trabajo La Fuerza del Derecho propone un derecho reflexivo, para hacerle contrapeso al sustrato del derecho y la emancipación de la colectividad normada en algunos aspectos. 
La anterior idea está en concordancia con el fenómeno de la Autopoieisis: ese derecho Reflexivo es una característica del derecho como sistema autopoiético, es decir, como un sistema completamente cerrado operacionalmente en la sociedad, esto es, circular y autorreferencial, donde no existen entradas ni salidas.

Además, el derecho al ser autopoiético tiene sus mecanismos de evolución, por lo tanto, no necesariamente marcha, siguiendo los pasos, con la evolución de la sociedad [8] [9].

\section{Conclusiones}

Después de agotarse toda la temática, se concluye:

1. El derecho, al igual que otras creaciones culturales, son fenómenos lingüísticos que se avientan al mundo, aunque en el plano ontológico no existen, y que, para el caso del derecho, los fundamentos de la comunicación social (Proceso, elemento, estructura e identidad) le dan forma a este, y al darle forma lo constituyen como un fenómeno de creación autopoiética.

2. La autopoiesis o creación autopoíetica consiste en que los componentes de un sistema constituyen con sus interacciones la red de producción que los origina, y bajo la óptica del derecho como producto cultural, la norma jurídica se transforma en un artefacto lingüístico: la norma jurídica ya no es quien crea el derecho sino que el permanente curso de comunicaciones dentro del sistema es lo que crea los estados transitorios de condensación de lo jurídico, tales como la Constitución, la ley, el contrato, etc.

3. Gracias a las aportaciones del profesor Teubner se asimila que es el derecho el que, como sujeto epistémico autónomo, construye su propia realidad social y que, por lo tanto, crea actores humanos como artefactos semánticos a través de sus operaciones jurídicas.

4. Frente al problema entre autonomía y heteronomía normativa, que tiene como sustento el hecho de que el discurso jurídico es a su vez un discurso social, el derecho debe abandonar el propósito de captar la autonomía de la constitución de realidades y procurar delegar esa atribución a otros discursos sociales.

5. Teubner concibe el derecho si bien cerrado y autopoiético, también orientado reflexivamente hacia la autonomía y la coordinación sistémica, con sus propios mecanismos evolutivos y que, por tanto, no necesariamente son los mismos que de los demás sistemas sociales.

Referencias

[1] B. Ramírez, La Teoría de Sistemas en el análisis del Estado, Cartagena: Universidad de Cartagena, 2013

[2] A. Henríquez, "Derecho y Autopoiesis", Revista de Derecho, p. 35, 2010

[3] M. Neves, "De la Autopoiesis a la Alopoiesis del Derecho", Doxa, p. 45, 1996

[4] G. Teubner, "ElDerecho como sujeto epistémico: Hacia una epistemología constructivista del Derecho", Doxa, p. 72, 1997

[5] K.M.E. y. E.A. Gamero, "El enfoque postkeynesiano a la microeconomía: una visión alternativa", Aglala. vol. 7, no. 1, 2016

[6] D. Mercado, Manual de Teoría Constitucional, Cartagena: Rodríguez Quito Editores, 2008

[7] F. Suarez-Vargas, "Aplicabilidad de las obligaciones por palabra en el derecho civil colombiano", Mundo Fesc, vol. 6, n. ${ }^{\circ}$ 11, pp. 27-32, sep. 2016, 2016 
El derecho como creación autopoética. análisis desde la perspectiva de Günther Teubner

\section{4}

[8] A. Squella, "Una Descripción del Derecho", Isonomía, vol. 27, pp. 51-70, 2007.

[9] J. Nuñes, "Modelos constitucionales en el ocaso", Revista de derecho. Universidad del Norte, no. 46, 2016 